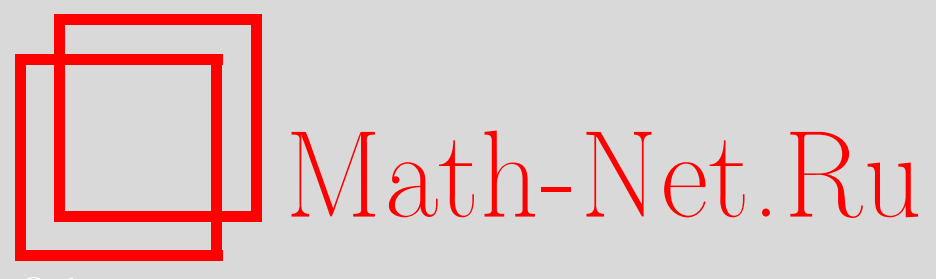

С. Арутюнян, Точка Нагеля и разностный треугольник, Квант, 2019, номер 9, 30-33

DOI: https://doi.org/10.4213/kvant20190905

Использование Общероссийского математического портала Math-Net.Ru подразумевает, что вы прочитали и согласны с пользовательским соглашением http://www.mathnet.ru/rus/agreement

Параметры загрузки:

IP : 54.224.135.184

26 апреля 2023 г., 02:16:20






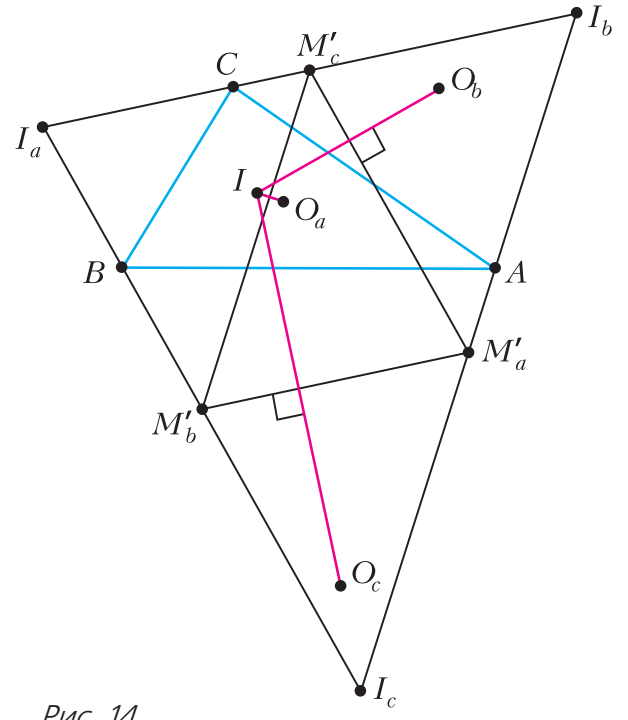

Pис. 14

$A B C$ является ортотреугольником в треугольнике $I_{a} I_{b} I_{c}$, а $\Delta M_{a}^{\prime} M_{b}^{\prime} M_{c}^{\prime}$ - середин- ным треугольником; это известные факты. Поэтому $M_{b}^{\prime} M_{c}^{\prime}$ - серединный перпендикуляр к отрезку $A I_{a}$. Получается, что точки $O_{a}$ и $I$ симметричны относительно прямой $M_{b}^{\prime} M_{c}^{\prime}$ (рис.14). Аналогичные рассуждения верны для пар точек $O_{b}, I$ и $O_{c}, I$.

Применяя лемму 3 для треугольника $M_{a}^{\prime} M_{b}^{\prime} M_{c}^{\prime}$ и точки $I$, получаем, что описанные окружности треугольников $O_{a} M_{c}^{\prime} O_{b}$, $O_{b} M_{a}^{\prime} O_{c}$ и $O_{c} M_{b}^{\prime} O_{a}$ пересекаются в одной точке на описанной окружности треугольника $M_{a}^{\prime} M_{b}^{\prime} M_{c}^{\prime}$. Однако эти окружности в точности являются окружностями пяти точек треугольника $A B C$, а описанная окружность треугольника $M_{a}^{\prime} M_{b}^{\prime} M_{c}^{\prime}$ является описанной окружностью треугольника $A B C . \mathrm{y}_{\mathrm{T}}$ верждение доказано.

В заключение автор выражает искреннюю благодарность А.И.Перегудину и П.А.Кожевникову за плодотворное обсуждение материалов настоящей статьи и ценные замечания по ее содержанию.

\section{Точка Нагеля и разностный треугольник}

\section{С.АРУТЮНЯН}

ЭТОЙ СТАТЬЕ РЕЧЬ ПОЙДЕТ ОБ ОДНОЙ

замечательной точке треугольника - точке Нагеля. Это точка пересечения отрезков, соединяющих вершины треугольника с точками касания противоположных сторон с вневписанными окружностями. С точкой Нагеля связано много красивых геометрических фактов. Упомянем известный факт о прямой Нагеля, которым мы будем пользоваться:

Для любого треугольника чентр вписанной окружности I, точка Нагеля $N$ и точка $M$ пересечения медиан лежат на одной прямой, при этом $\overrightarrow{M N}=2 \overrightarrow{I M}$.

Немного по-другому этот факт можно переформулировать так. Пусть $M_{a}, M_{b}, M_{c}$

DOI: https://doi.org/10.4213/kvant20190905
- середины соответствующих сторон треугольника. Тогда при гомотетии с центром в $M$ и коэффициентом $-1 / 2$ треугольник $A B C$ переходит в $\Delta M_{a} M_{b} M_{c}$, а точка $N-$ в точку $I$, т.е. I является точкой Нагеля для серединного треугольника $M_{a} M_{b} M_{c}$.

А теперь посмотрим на один замечательный класс треугольников - так называемые разностные треугольники. Разностным называют треугольник, длины сторон которого составляют арифметическую прогрессию. У класса разностных треугольников существует много других красивых эквивалентных определений. Этому классу, например, посвящен параграф книги А.Д.Блинкова «Классические средние в арифметике и геометрии» (М.: МЦНМО, 2016). Упомянем два условия, эквивалентных условию разностности (при этом ограничимся рассмотрением только неравнобедренных треугольников).

Итак, пусть $A B C$ - неравнобедренный треугольник, $a=B C, b=C A, c=A B$. Тогда следующие условия эквивалентны:

(1) $a+c=2 b$ (т.е. треугольник разностный, со средней по длине стороной $b$ );

(2) точка I равноудалена от $M_{a} u M_{c}$ ( puc.1);

(3) четьрехугольник $B M_{a} I M_{c}-$ вписанный. 


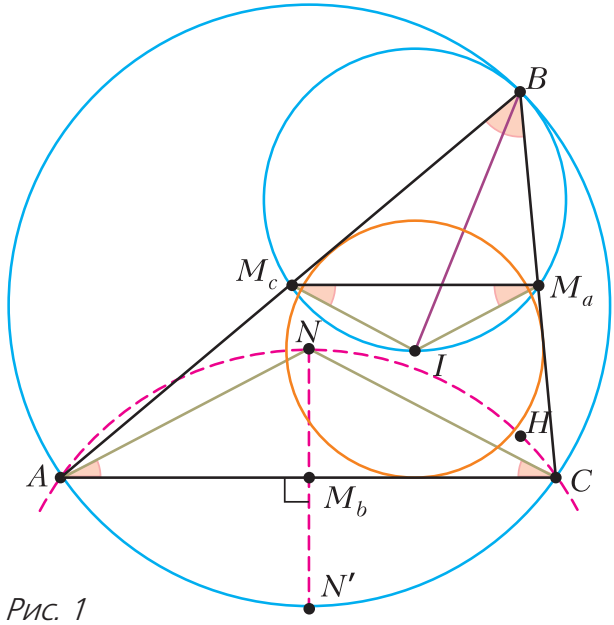

Условие (3), очевидно, эквивалентно условию на углы

( $\left.3^{\prime}\right) \angle M_{a} I M_{c}=180^{\circ}-\angle A B C$.

Попробуем получить условия на треугольник, эквивалентные приведенным, но связанные с точкой Нагеля.

Рассмотренная выше гомотетия переводит треугольник $A N C$ в $\Delta M_{a} I M_{c}$. А это значит, что условие (2) эквивалентно условию

(4) точка $N$ равноудалена от $A$ и $C$.

Да, оказывается, из равенства $N A=N C$ совершенно не следует, что треугольник равнобедренный! Неожиданно, но красиво!

Уже неплохо. Попробуем поработать с другими условиями.

Все та же гомотетия помогает переформулировать условие ( $3^{\prime}$ ) как

(5) $\angle A N C=180^{\circ}-\angle A B C$.

Рассмотрим точку $N^{\prime}$, симметричную $N$ относительно $A C$, так что $\angle A N C=\angle A N^{\prime} C$. Условие (5) эквивалентно тому, что $\angle A N^{\prime} C+\angle A B C=180^{\circ}$, т.е. тому, что четырехугольник $A B C N^{\prime}$ вписанный, или условию

(5' ) $N^{\prime}$ лежит на описанной окружности треугольника $A B C$.

Как известно, точка, симметричная ортоцентру $Н$ относительно прямой, содержащей сторону треугольника, лежит на описанной окружности. Отражение относительно $A C$ дает возможность теперь переформулировать условие $\left(5^{\prime}\right)$ как

( $\left.5^{\prime \prime}\right)$ точки $A, C, N, H$ лежат на одной окружности .

Заметим, что из условия ( $\left.5^{\prime}\right)$ следует (4), значит, $A N^{\prime}=C N^{\prime}$, т.е. $N^{\prime}-$ середина дуги.
Получается, что ( $\left.5^{\prime}\right)$ эквивалентно формально более слабому факту

$\left(5^{\prime \prime \prime}\right) N^{\prime}$ является серединой дуги $A C$ (не содержащей точки $B$ ).

Видим, что в разностном треугольнике $N^{\prime}$ лежит на биссектрисе угла $B$. Этот факт можно переформулировать в такой занятной форме. Пусть имеется бильярд в форме треугольника $A B C$, в котором $a+c=2 b$. Тогда бильярдный шар, выпущенный из вершины $B$ вдоль биссектрисы угла $A B C$, после отражения относительно борта $A C$ пройдет через точку Нагеля. Предлагаем читателю подумать, верно ли утверждение, обратное данному.

Можно продолжать и получать другие условия, в том числе и не использующие точку $N$. Например, при помощи изогонального сопряжения условие ( $\left.5^{\prime \prime}\right)$ можно переформулировать так.

Пусть $S$ - центр гомотетии с положительным коэффициентом, переводящей вписанную окружность в описанную. Тогда условие ( $5^{\prime \prime}$ ) эквивалентно тому, что точки $A, C, O$, $S$ лежат на одной окружности (рис.2). Секрет здесь в том, что $O$ и $H$, а также $S$ и $N$ - пары изогонально сопряженных точек; а кроме того, если точки $A, C, X, Y$ лежат на одной окружности, то же верно и для точек $A, C, X_{1}, Y_{1}$, где $X_{1}$ и $Y_{1}$ - точки, изогонально сопряженные точкам $X$ и $Y$. (Докажите это!)

Но вернемся к условию (5). Продлим отрезки $A N$ и $C N$ до нагелиан $A N_{a}$ и $C N_{a}$. Условие (5) приводим к виду

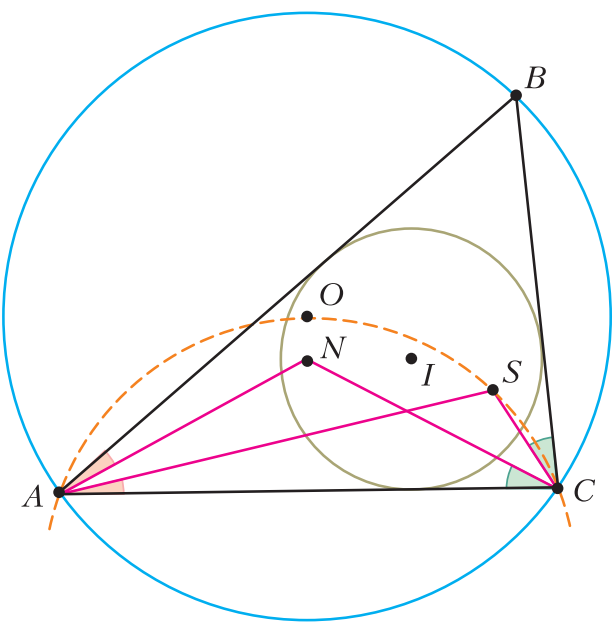

PUC. 2 
$\angle N_{a} N N_{c}=180^{\circ}-\angle N_{a} B N_{c}$. А это эквивалентно тому, что

(6) точки $B, N_{a}, N, N_{c}$ лежат на одной окружности (рис. 3).

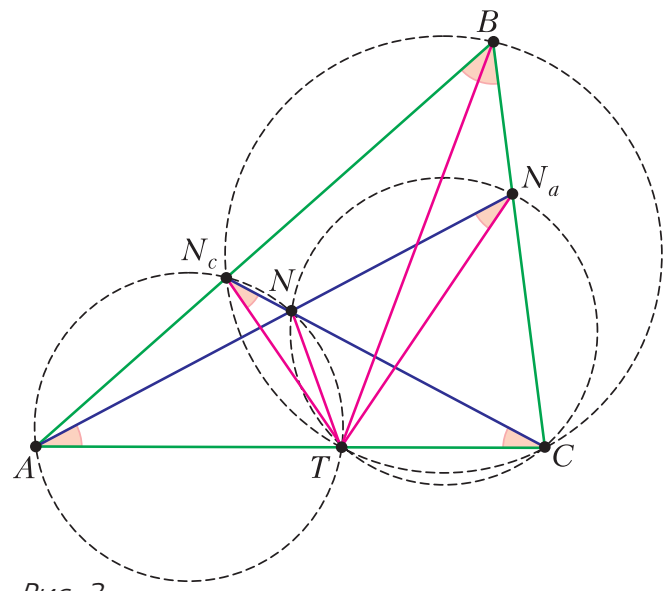

Рис. 3

Проведем окружность $C N N_{a}$ и найдем точку $T$ ее пересечения с $A C$. Тогда $\angle N T A=\angle N N_{a} C$. Условие (6) эквивалентно равенству

$$
\angle N N_{c} B=\angle N N_{a} C \Leftrightarrow \angle N N_{c} B=\angle N T A,
$$

следовательно, точки $A, N_{c}, N, T$ лежат на одной окружности. Иными словами, (6) эквивалентно тому, что окружности $C N N_{a}$ и $A N N_{c}$ пересекаются на $A C$.

В разностном треугольнике, с учетом условия (4), получаем

$$
\angle C N_{c} T=\angle N A C=\angle N C A=\angle A N_{a} T .
$$

Тогда треугольники $A T N_{a}$ и $C T N_{c}$ равнобедренные и $T$ - точка пересечения серединных перпендикуляров к нагелианам $A N_{a}$ и $C N_{c}$. (Подумайте, следует ли из того, что серединные перпендикуляры к нагелианам $A N_{a}$ и $C N_{c}$ пересекаются на $A C$, что треугольник $A B C$ разностный.)

Продолжаем рассуждения для разностного треугольника $A B C$. Треугольники $T C N_{a}$ и $T N_{c} A$ равны (получаются один из другого поворотом вокруг точки $T$ ), значит, $T$ равноудалена от прямых $A B$ и $B C$, т.е. $T$ является основанием биссектрисы!

В завершение обсудим ситуацию, когда отрезок $B D$ делит треугольник $A B C$ на два разностных треугольника $A B D$ и $C B D$ со средней стороной $B D$ (рис.4). Нетрудно понять (сделайте это), что в таком случае

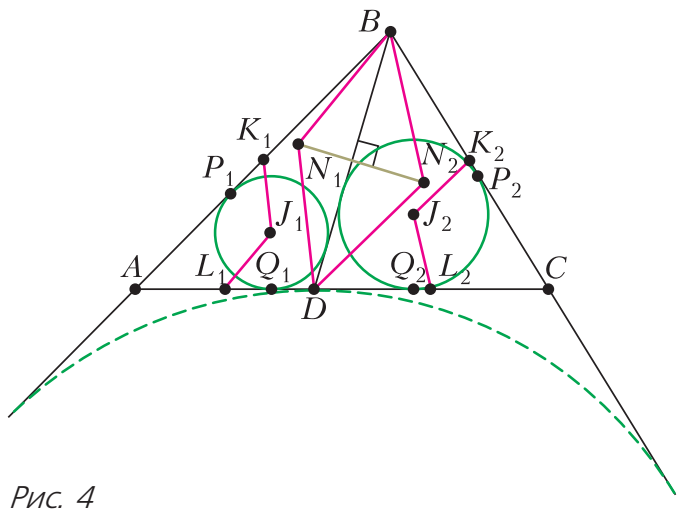

$B D$ обязана быть нагелианой $B N_{b}$, и при этом $B N_{b}=p / 2$, т.е. нагелиана равна четверти периметра. Если это условие выполнено, то точки Нагеля $N_{1}$ и $N_{2}$ треугольников $A B D$ и $C B D$ равноудалены от точек $B$ и $D$, откуда $B D \perp N_{1} N_{2}$.

Зададимся вопросом - верно ли обратное? Следует ли из условия $B D \perp N_{1} N_{2}$ то, что $B D=p / 2$ ? Оказывается, ответ на этот вопрос положительный. Докажем это.

Впишем в треугольники $A B D$ и $C B D$ окружности с центрами $J_{1}$ и $J_{2}$ и отметим точки $P_{1}, Q_{1}, Q_{2}, P_{2}$ их касания с отрезками $B A, A D, D C, C B$ соответственно. Также обозначим через $K_{1}, L_{1}, L_{2}, K_{2}$ середины отрезков $B A, A D, D C, C B$ соответственно.

Для того чтобы имело место $N_{1} N_{2} \perp B D$, необходимо и достаточно, чтобы $N_{1} B^{2}-N_{1} D^{2}=N_{2} B^{2}-N_{2} D^{2} \quad$ (проверьте это). Рассматривая гомотетию, упомянутую в доказательстве условия (4), получаем, что $D N_{1}=2 K_{1} J_{1}$ и $B N_{1}=2 L_{1} J_{1}, D N_{2}=2 K_{2} J_{2}$ и $B N_{2}=2 L_{2} J_{2}$. Отсюда

$$
\begin{aligned}
N_{1} B^{2}- & N_{1} D^{2}=N_{2} B^{2}-N_{2} D^{2} \Leftrightarrow \\
\Leftrightarrow & J_{1} L_{1}^{2}-J_{1} K_{1}^{2}=J_{2} L_{2}^{2}-J_{2} K_{2}^{2} \Leftrightarrow \\
\Leftrightarrow & \left(J_{1} Q_{1}^{2}+Q_{1} L_{1}^{2}\right)-\left(J_{1} P_{1}^{2}+P_{1} K_{1}^{2}\right)= \\
= & \left(J_{2} Q_{2}^{2}+Q_{2} L_{2}^{2}\right)-\left(J_{2} P_{2}^{2}+P_{2} K_{2}^{2}\right) \Leftrightarrow \\
\Leftrightarrow & Q_{1} L_{1}^{2}-P_{1} K_{1}^{2}=Q_{2} L_{2}^{2}-P_{2} K_{2}^{2} \Leftrightarrow \\
\Leftrightarrow & (A B-B D)^{2}-(A D-B D)^{2}= \\
= & (C B-B D)^{2}-(C D-B D)^{2} \Leftrightarrow \\
& \Leftrightarrow B D=\frac{A B^{2}-B C^{2}+C D^{2}-A D^{2}}{2(A B-B C+C D-A D)} .
\end{aligned}
$$

Подставив $A B=c, B C=a, A D=p-c$, 
$C D=p-a$, получаем

$$
B D=\frac{c^{2}-a^{2}+(p-a)^{2}-(p-c)^{2}}{2(c-a+(p-a)-(p-c))}=\frac{p}{2} .
$$

Нетрудно заметить, что проведенные выше рассуждения не изменяются, если «вырожденный четырехугольник» $A B C D$ заменить на любой другой четырехугольник, в котором $B A+A D=B C+C D$ (для таких четырехугольников, как и для описанных, существует окружность, касающаяся всех прямых, содержащих их стороны).
Завершая наш рассказ, отметим, что есть несколько других интересных свойств, связывающих точку Нагеля с треугольниками, в которых отношение суммы длин двух сторон к длине третьей стороны равно некоторому фиксированному числу. Треугольники, для которых это отношение равно 3, хорошо исследованы и про них можно прочитать в статье А.Заславского «Приключения одной задачи» в «Кванте» №12 за 2017 год. Читатель может самостоятельно попробовать исследовать свойства таких классов треугольников и связать их с точкой Нагеля.

\section{КОНКУРС ИМЕНИ А.П.САВИНА}

Мы начинаем очередной конкурс по решению математических задач. Они рассчитаны в первую очередь на учащихся 7-9 классов, но мы будем рады участию школьников всех возрастов. Конкурс проводится совместно с журналом “Квантик».

Высылайте решения задач, с которыми справитесь, электронной почтой по адресу: savin.contest@gmail.com или обычной почтой по адресу: 119296 Москва, Ленинский проспект, 64-А, "Квант" (с пометкой "Конкурс имени А.П.Савина"). Кроме имени и фамилии укажите город, школу и класс, в котором вы учитесь, а также обратный почтовый адрес.

Мы приветствуем участие в конкурсе не только отдельных школьников, но и команд (в таком случае присылается одна работа со списком участников). Участвовать можно, начиная с любого тура. Победителей ждут дипломы журнала “Квант» и призы. Задания, решения и результаты публикуются на сайте sites.google.com/view/savin-contest Желаем успеха!

1. Вася хочет выбрать среди чисел а) 1, $2, \ldots, 50 ;$ б) 1, 2, ., 51 как можно больше чисел так, чтобы любые два числа различались хотя бы на 3. Сколько способов сделать это есть у Васи?

\section{П.Кожевников}

2. Квантик загадал целое число от 0 до 99, а Ноутик его отгадывает. Число считается отгаданным, если Ноутик его назвал. За ход Ноутик называет четыре целых числа от 0 до 99, обладающих одним из двух свойств: либо первый разряд у всех чисел совпадает, а вторые разряды последовательные, либо второй разряд у всех чисел совпадает, а первые разряды последовательные (у однозначных чисел первый разряд считаем равным 0). Например, наборы 13, 14, 15, 16 и 3, 13, 23, 33 подходят, а набор 18, 19, 20, 21 - нет. За какое минимальное чис- ло ходов Ноутик может гарантированно отгадать число?

Ю.Маркелов, С.Маркелов

3. Дан отрезок $a$ и правильный треугольник со стороной $b$. Постройте (циркулем и линейкой) внутри этого треугольника правильный треугольник со стороной $a$ такой, чтобы вершины внешнего треугольника лежали на продолжениях сторон внутреннего.

\section{Е.Бакаев}

4. Верно ли, что числа вида $1+x+$ $+x^{2017}+x^{2018}+x^{2019}$ являются составными для всех натуральных $x>1$ ?

B.Pacmopryeв 